\title{
Weighted Ratio-cum-Product Estimator for Finite Population Mean
}

\author{
K.B. Panda ${ }^{1 *}$ and M. Sen ${ }^{2}$ \\ ${ }^{1,2}$ P.G. Department of Statistics, Utkal University, Bhubaneswar, Odisha-751004 \\ Corresponding Author: kunja.st@utkaluniversity.ac.in
}

Available online at: www.isroset.org

Accepted 18/Aug/2018, Online 30/Aug/2018

\begin{abstract}
We have, in this paper, proposed a new ratio-cum-product estimator of finite population mean using information on two auxiliary variates. The bias and mean squared error of the proposed estimator, up to the first order of approximation have been derived. The proposed estimator, under optimal weights, is shown to be superior to the competing estimators. Empirical investigations have been carried out in support of the theoretical findings.
\end{abstract}

Keywords: Auxiliary variable, Ratio-cum-product estimator

\section{INTRODUCTION}

In survey sampling, a considerable attention is given for improving upon the usual unbiased estimator by the use of supplementary variable(s) in sampling theory and practice for estimation of population characteristics. The literature on survey sampling describes agreement of various techniques for utilizing information on auxiliary variate by ratio, product and regression methods of estimation to estimate the population parameters, out of which ratio and product are being easily obtainable and are more prevalent in practice.

Over the years, various estimators have been developed in simple random sampling to estimate the population characters using auxiliary information. Some noteworthy contributions in this area have been made by several authors including Hansen(1953), Olikin(1958), Goodman(1960), Koop(1964), Murthy(1964), Singh(1965), Singh(1966), Singh (1967) and many others.

In the present paper, we have studied some of the existing estimators for the population mean of a study variate by utilizing information on two auxiliary variates of which one is positively correlated with the study variate, while the other is negatively correlated. The proposed weighted ratio-cum-product estimator performs better than Singh's estimator, usual ratio and product estimators and simple unbiased estimatorunder practical conditions.
We consider a finite population of size $\mathrm{N}$, arbitrarily labelled 1, 2...N. Let $\mathrm{Y}$ and $\left(\mathrm{X}_{1}, \mathrm{X}_{2}\right)$ be the study and auxiliary variates, respectively, where $\mathrm{X}_{1}$ is positively correlated with $\mathrm{Y}$, while $\mathrm{X}_{2}$ is negatively correlated with Y. Assuming that the population means $\bar{X}_{1}$ and $\bar{X}_{2}$ are known, a sample of size $n$ (with $n<N$ ) is drawn from the population size $\mathrm{N}$ using simple random sampling without replacement (SRSWOR) scheme to estimate the population mean $\bar{Y}=\frac{\sum_{i=1}^{N} Y_{i}}{N}$ of the study variate.

The whole paper is composed of six sections followed by references and authors profile. Section-I contains noteworthy contributions by several authors over the years to estimate population characters using auxiliary information. Section-II describes briefly some existing estimators by different authors. In section-III, the expressions of bias and mean square error of the proposed estimator have been derived, and also expression for optimum weight has been arrived at. Section-IV discusses efficiency comparisons of the proposed estimator with respect to competing estimators. Section-V deals with the empirical study to justify the supremacy of theoretical findings. Section-VI presents the summary and future directions.

\section{REVIEW OF LITERATURE OF EXISTING ESTIMATORS}

The variance of simple unbiased estimator is given by 


$$
V(\bar{y})=\theta \bar{Y}^{2} C_{y}^{2}
$$

The usual ratio and product estimators of $\bar{Y}$ are, respectively,

$$
\bar{y}_{R}=\frac{\bar{y}}{\bar{x}} \bar{X}
$$

and

$$
\bar{y}_{P}=\frac{\bar{y}}{\bar{x}} \bar{x},
$$

whose biases and mean square errors, up to first degree of approximation, are, respectively,

$$
\begin{gathered}
B\left(\bar{y}_{R}\right)=\theta \bar{Y}(1-C) C_{x}^{2}, \\
B\left(\bar{y}_{P}\right)=\theta \bar{Y} C C_{x}^{2}, \\
M S E\left(\bar{y}_{R}\right)=\theta \bar{Y}^{2}\left\{C_{y}^{2}+(1-2 C) C_{x}^{2}\right\}
\end{gathered}
$$

and

$$
\operatorname{MSE}\left(\bar{y}_{P}\right)=\theta \bar{Y}^{2}\left\{C_{y}^{2}+(1+2 C) C_{x}^{2}\right\}
$$

where $C_{x}$ and $C_{y}$ are the coefficients of variations of $x$ and $y$, respectively, $C=\rho \frac{C_{y}}{C_{x}}$ and $f=\frac{n}{N}$.

M.P. Singh (1967) has suggested ratio-cum-product estimator, which is given by

$$
\bar{y}_{R P}=\bar{y}\left(\frac{\bar{x}_{1}}{\bar{x}_{1}}\right)\left(\frac{\bar{x}_{2}}{\bar{x}_{2}}\right)
$$

The bias and mean square error up to first degree of approximation are, respectively,

$$
\begin{gathered}
B\left(\bar{y}_{R P}\right)=\theta \bar{Y}\left(C_{1}^{2}+C_{02}-C_{01}-C_{12}\right) \quad \text { (9) and } \\
M S E\left(\bar{y}_{R P}\right)=\theta \bar{Y}^{2}\left(\begin{array}{c}
\left(C_{0}^{2}+C_{1}^{2}+C_{2}^{2}-2 C_{01}-2 C_{12}+\right. \\
\left.2 C_{02}\right) .
\end{array}\right.
\end{gathered}
$$

\section{PROPOSED WEIGHTED RATIO-CUM- PRODUCT ESTIMATOR}

We propose a weighted ratio-cum-product estimator for estimating the population mean $\bar{Y}$, which is given by

$$
\bar{y}_{R P}^{*}=\bar{y}\left(W_{1} \frac{\bar{x}_{1}}{\bar{x}_{1}}+W_{2} \frac{\bar{x}_{2}}{\bar{x}_{2}}\right)
$$

$$
\Rightarrow \bar{y}=\bar{Y}\left(1+\varepsilon_{0}\right),
$$

$\varepsilon_{1}=\frac{\bar{x}_{1}-\bar{X}_{1}}{\bar{X}_{1}}$

$\Rightarrow \bar{x}_{1}=\bar{X}_{1}\left(1+\varepsilon_{1}\right)$ and

$\varepsilon_{2}=\frac{\overline{\mathrm{x}}_{2}-\overline{\mathrm{X}}_{2}}{\overline{\mathrm{X}}_{2}}$

$\Rightarrow \bar{x}_{2}=\bar{X}_{2}\left(1+\varepsilon_{2}\right)$,

Now, $\mathrm{E}\left(\varepsilon_{0}\right)=\mathrm{E}\left(\varepsilon_{1}\right)=\mathrm{E}\left(\varepsilon_{2}\right)=0$,

$\mathrm{E}\left(\varepsilon_{0}^{2}\right)=\theta \mathrm{C}_{\mathrm{y}}^{2}, \mathrm{E}\left(\varepsilon_{1}^{2}\right)=\theta \mathrm{C}_{\mathrm{x}_{1}}^{2}, \mathrm{E}\left(\varepsilon_{2}^{2}\right)=\theta \mathrm{C}_{\mathrm{x}_{2}}^{2}$

$\mathrm{E}\left(\varepsilon_{0} \varepsilon_{1}\right)=\theta \rho \mathrm{C}_{\mathrm{y}} \mathrm{C}_{\mathrm{x}_{1}}, \mathrm{E}\left(\varepsilon_{1} \varepsilon_{2}\right)=\theta \rho \mathrm{C}_{\mathrm{x}_{1}} \mathrm{C}_{\mathrm{x}_{2}}$,

$\mathrm{E}\left(\varepsilon_{0} \varepsilon_{2}\right)=\theta \rho \mathrm{C}_{\mathrm{y}} \mathrm{C}_{\mathrm{x}_{2}}$, where $\theta=\frac{1-\mathrm{f}}{\mathrm{n}}$

Putting the values of $\bar{y}, \bar{x}_{1}$ and $\bar{x}_{2}$ in the expression-(11), we get

$$
\bar{y}_{R P}^{*}=\bar{Y}\left(1+\varepsilon_{0}\right)\left(W_{1} \frac{\bar{X}_{1}}{\bar{X}_{1}\left(1+\varepsilon_{1}\right)}+W_{2} \frac{\bar{X}_{2}\left(1+\varepsilon_{2}\right)}{\bar{X}_{2}}\right)
$$

Upon simplification, we find that

$$
\begin{array}{r}
\bar{y}_{R P}^{*}=\bar{Y}\left(1-W_{1} \varepsilon_{1}+W_{2} \varepsilon_{2}+W_{1} \varepsilon_{1}^{2}+\varepsilon_{0}-W_{1} \varepsilon_{0} \varepsilon_{1}+\right. \\
\left.W_{2} \varepsilon_{0} \varepsilon_{2}\right)
\end{array}
$$

The bias of the proposed estimator, up to the first degree of approximation, is

$$
B\left(\bar{y}_{R P}^{*}\right)=\mathrm{E}\left(\bar{y}_{R P}^{*}\right)-\bar{Y}
$$

$$
\begin{array}{r}
=\bar{Y} E\left(-W_{1} \varepsilon_{1}+W_{2} \varepsilon_{2}+W_{1} \varepsilon_{1}^{2}+\varepsilon_{0}-W_{1} \varepsilon_{0} \varepsilon_{1}+\right. \\
\left.W_{2} \varepsilon_{0} \varepsilon_{2}\right)
\end{array}
$$

$=\theta \bar{Y}\left(W_{1} C_{1}^{2}-W_{1} C_{01}+W_{2} C_{02}\right)$

Similarly, the mean square error, to the first degree of approximation, is

$$
\begin{aligned}
& M\left(\bar{y}_{R P}^{*}\right)=\mathrm{E}\left[\bar{y}_{R P}^{*}-\bar{Y}\right]^{2} \\
& =\bar{Y} E\left[-W_{1} \varepsilon_{1}+W_{2} \varepsilon_{2}+W_{1} \varepsilon_{1}^{2}+\varepsilon_{0}-\begin{array}{r}
W_{1} \varepsilon_{0} \varepsilon_{1}+ \\
\left.W_{2} \varepsilon_{0} \varepsilon_{2}\right]^{2}
\end{array}\right.
\end{aligned}
$$

Let $\varepsilon_{0}=\frac{\bar{y}-\bar{Y}}{\bar{Y}}$ 


$$
\begin{aligned}
& =\theta \bar{Y}^{2} \\
& \left(W_{1}^{2} C_{1}^{2}+W_{2}^{2} C_{2}^{2}+C_{0}^{2}-2 W_{1} C_{01}+\right. \\
& \left.2 W_{2} C_{02}-2 W_{1} W_{2} C_{12}\right) .
\end{aligned}
$$

With a view to determining the most suitable value of $W_{1}$, (and thus $W_{2}$ ), we proceed to minimize the mean square error subject to the variation in $W_{1}$, implying thereby that

$$
\begin{gathered}
\frac{\partial M S E\left(\bar{y}_{R P}^{*}\right)}{\partial W_{1}}=0 \\
\Rightarrow \theta \bar{Y}^{2}\left(2 W_{1} C_{1}^{2}-2\left(1-W_{1}\right) C_{2}^{2}-2 C_{01}-2 C_{02}-\right. \\
\left.2 C_{12}+4 W_{1} C_{12}\right)=0 \\
\Rightarrow W_{1}=\frac{C_{2}^{2}+C_{01}+C_{02}+C_{12}}{C_{1}^{2}+C_{2}^{2}+2 C_{12}}=W_{1 o p t}=1-W_{2 o p t}, \text { say. }
\end{gathered}
$$

\section{EFFICIENCY COMPARISON}

On comparison of (14) under optimum weights, with (10), we get

$$
\begin{aligned}
& M\left(\bar{y}_{R P}^{*}\right)-M\left(\bar{y}_{R P}\right)<0 \\
& \Rightarrow \theta \bar{Y}^{2}\left(W_{1}^{2} C_{1}^{2}+W_{2}^{2} C_{2}^{2}+C_{0}^{2}-2 W_{1} C_{01}+2 W_{2} C_{02}-\right. \\
& \left.2 W_{1} W_{2} C_{12}\right)-\theta \bar{Y}^{2}\left(C_{0}^{2}+C_{1}^{2}+C_{2}^{2}-2 C_{01}-2 C_{12}+\right. \\
& \left.2 C_{02}\right)<0 \\
& \Rightarrow \theta \bar{Y}^{2}\left(W_{1}^{2} C_{1}^{2}+W_{2}^{2} C_{2}^{2}+C_{0}^{2}-2 W_{1} C_{01}+2 W_{2} C_{02}-\right. \\
& \left.2 W_{1} W_{2} C_{12}-C_{0}^{2}-C_{1}^{2}-C_{2}^{2}+2 C_{01}+2 C_{12}-2 C_{02}\right)<0 \\
& \Rightarrow W_{1}^{2} C_{1}^{2}+W_{2}^{2} C_{2}^{2}-2 W_{1} C_{01}+2 W_{2} C_{02}-2 W_{1} W_{2} C_{12}- \\
& C_{1}^{2}-C_{2}^{2}+2 C_{01}+2 C_{12}-2 C_{02}<0 \\
& \Rightarrow W_{1}^{2} C_{1}^{2}+\left(1-W_{1}\right)^{2} C_{2}^{2}-2 W_{1} C_{01}+2\left(1-W_{1}\right) C_{02}- \\
& 2 W_{1}\left(1-W_{1}\right) C_{12}-C_{1}^{2}-C_{2}^{2}+2 C_{01}+2 C_{12}-2 C_{02}<0 \\
& \Rightarrow W_{1}^{2} C_{1}^{2}+C_{2}^{2}+W_{1}^{2} C_{2}^{2}-2 W_{1} C_{2}^{2}-2 W_{1} C_{01}+2 C_{02}- \\
& 2 W_{1} C_{02}-2 W_{1} C_{12}+2 W_{1}^{2} C_{12}-C_{1}^{2}-C_{2}^{2}+2 C_{01}+ \\
& 2 C_{12}-2 C_{02}<0 \\
& \Rightarrow W_{1}^{2} C_{1}^{2}+W_{1}^{2} C_{2}^{2}-2 W_{1} C_{2}^{2}-2 W_{1} C_{01}-2 W_{1} C_{02}- \\
& 2 W_{1} C_{12}+2 W_{1}^{2} C_{12}-C_{1}^{2}+2 C_{01}+2 C_{12}<0 \\
& \Rightarrow W_{1}^{2} C_{1}^{2}+W_{1}^{2} C_{2}^{2}-2 W_{1} C_{2}^{2}-2 W_{1} C_{01}-2 W_{1} C_{02}- \\
& 2 W_{1} C_{12}+2 W_{1}^{2} C_{12}<C_{1}^{2}-2 C_{01}-2 C_{12} \\
& \Rightarrow W_{1}^{2}\left(C_{1}^{2}+C_{2}^{2}+2 C_{12}\right)-2 W_{1}\left(C_{2}^{2}+C_{01}+C_{02}+C_{12}\right)< \\
& C_{1}^{2}-2 C_{01}-2 C_{12}
\end{aligned}
$$

$$
\begin{aligned}
& \Rightarrow \frac{\left(C_{2}^{2}+C_{01}+C_{02}+C_{12}\right)^{2}}{\left(C_{1}^{2}+C_{2}^{2}+2 C_{12}\right)^{2}}\left(C_{1}^{2}+C_{2}^{2}+2 C_{12}\right)- \\
& 2\left(\frac{C_{2}^{2}+C_{01}+C_{02}+C_{12}}{C_{1}^{2}+C_{2}^{2}+2 C_{12}}\right)\left(C_{2}^{2}+C_{01}+C_{02}+C_{12}\right) \\
& <C_{1}^{2}-2 C_{01}-2 C_{12} \\
& \Rightarrow \frac{\left(C_{2}^{2}+C_{01}+C_{02}+C_{12}\right)^{2}}{\left(C_{1}^{2}+C_{2}^{2}+2 C_{12}\right)}-2 \frac{\left(C_{2}^{2}+C_{01}+C_{02}+C_{12}\right)}{\left(C_{1}^{2}+C_{2}^{2}+2 C_{12}\right)}\left(C_{2}^{2}+C_{01}+\right. \\
& \left.C_{02}+C_{12}\right)<C_{1}^{2}-2 C_{01}-2 C_{12} \\
& \Rightarrow \frac{\left(C_{2}^{2}+C_{01}+C_{02}+C_{12}\right)^{2}-2\left(C_{2}^{2}+C_{01}+C_{02}+C_{12}\right)\left(C_{2}^{2}+C_{01}+C_{02}+C_{12}\right)}{C_{1}^{2}+C_{2}^{2}+2 C_{12}} \\
& <C_{1}^{2}-2 C_{01}-2 C_{12} \\
& \Rightarrow\left(C_{2}^{2}+C_{01}+C_{02}+C_{12}\right)^{2}-2\left(C_{2}^{2}+C_{01}+C_{02}+\right. \\
& \left.C_{12}\right)\left(C_{2}^{2}+C_{01}+C_{02}+C_{12}\right)<\left(C_{1}^{2}+C_{2}^{2}+2 C_{12}\right)\left(C_{1}^{2}-\right. \\
& \left.2 C_{01}-2 C_{12}\right) \\
& \Rightarrow C_{2}^{4}+C_{01}^{2}+C_{02}^{2}+C_{12}^{2}+2 C_{2}^{2} C_{01}+2 C_{2}^{2} 02+2 C_{2}^{2} C_{12}+ \\
& 2 C_{01} C_{02}+2 C_{01} C_{12}+2 C_{02} C_{12}-2 C_{2}^{4}-2 C_{2}^{2} C_{01}- \\
& 2 C_{2}^{2} C_{02}-2 C_{2}^{2} C_{12}-2 C_{01} C_{2}^{2}-2 C_{01}^{2}-2 C_{01} C_{02}- \\
& 2 C_{01} C_{12}-2 C_{02} C_{2}^{2}-2 C_{02} C_{01}-2 C_{02}^{2}-2 C_{02} C_{12}- \\
& 2 C_{12} C_{2}^{2}-2 C_{12} C_{01}-2 C_{12} C_{02}-2 C_{12}^{2}<C_{1}^{4}-2 C_{1}^{2} C_{01}- \\
& 2 C_{1}^{2} C_{12}+C_{1}^{2} C_{2}^{2}-2 C_{01} C_{2}^{2}-2 C_{2}^{2} C_{12}+2 C_{1}^{2} C_{12}- \\
& 4 C_{01} C_{12}-4 C_{12}^{2} \\
& \Rightarrow-C_{2}^{4}-C_{01}^{2}-C_{02}^{2}-C_{12}^{2}-2 C_{02} C_{2}^{2}-2 C_{02} C_{01}- \\
& 2 C_{12} C_{2}^{2}-2 C_{12} C_{01}-2 C_{12} C_{02}<C_{1}^{4}-2 C_{1}^{2} C_{01}- \\
& 2 C_{1}^{2} C_{12}+C_{1}^{2} C_{2}^{2}-2 C_{2}^{2} C_{12}+2 C_{1}^{2} C_{12}-4 C_{01} C_{12}-4 C_{12}^{2} \\
& \Rightarrow-C_{2}^{4}-C_{01}^{2}-C_{02}^{2}-C_{12}^{2}-2 C_{02} C_{2}^{2}-2 C_{02} C_{01}- \\
& 2 C_{12} C_{01}-2 C_{12} C_{02}<C_{1}^{4}-2 C_{1}^{2} C_{01}-2 C_{1}^{2} C_{12}+C_{1}^{2} C_{2}^{2}+ \\
& 2 C_{1}^{2} C_{12}-4 C_{01} C_{12}-4 C_{12}^{2} \\
& \Rightarrow C_{2}^{4}+C_{01}^{2}+C_{02}^{2}+C_{12}^{2}+2 C_{02} C_{2}^{2}+2 C_{02} C_{01}+ \\
& 2 C_{12} C_{01}+2 C_{12} C_{01}>-C_{1}^{4}+2 C_{1}^{2} C_{01}+2 C_{1}^{2} C_{12}-C_{1}^{2} C_{2}^{2}- \\
& 2 C_{1}^{2} C_{12}+4 C_{01} C_{12}+4 C_{12}^{2} \\
& \Rightarrow C_{2}^{4}+C_{01}^{2}+C_{02}^{2}+C_{12}^{2}+2 C_{02} C_{2}^{2}+2 C_{02} C_{01}+ \\
& 2 C_{12} C_{01}+2 C_{12} C_{01}+C_{1}^{4}+C_{1}^{2} C_{2}^{2}+2 C_{1}^{2} C_{12}>2 C_{1}^{2} C_{01}+ \\
& 2 C_{1}^{2} C_{12}+4 C_{01} C_{12}+4 C_{12}^{2} \\
& \Rightarrow C_{2}^{4}+C_{01}^{2}+C_{02}^{2}+C_{12}^{2}+2 C_{02} C_{2}^{2}+2 C_{02} C_{01}+C_{1}^{4}+ \\
& C_{1}^{2} C_{2}^{2}>2 C_{1}^{2} C_{01}+4 C_{12}^{2} \\
& \Rightarrow\left(C_{01}+C_{02}\right)^{2}+C_{1}^{2}+C_{2}^{4}+C_{12}^{2}+C_{1}^{2} C_{2}^{2}+2 C_{02} C_{2}^{2}> \\
& 2 C_{1}^{2} C_{01}+4 C_{12}^{2} \\
& \Rightarrow\left(C_{01}+C_{02}\right)^{2}+C_{1}^{2}+C_{2}^{4}+C_{1}^{2} C_{2}^{2}+2 C_{02} C_{2}^{2}> \\
& 2 C_{1}^{2} C_{01}+3 C_{12}^{2}
\end{aligned}
$$


$\Rightarrow\left(C_{01}+C_{02}\right)^{2}+C_{1}^{2}\left(1+C_{2}^{2}\right)+C_{2}^{2}\left(1+2 C_{02}\right)>$

$2 C_{1}^{2} C_{01}+3 C_{12}^{2}$

Thus, the proposed weighted ratio-cum-product estimator fares better than the usual ratio-cum-product estimator if the above inequality holds good.

\section{EMPIRICAL STUDY}

\section{Example-1}

Source: Singh (1969)

Y: Number of females employed

$X_{1}$ : Number of females in service

$X_{2}$ :Number of educated females

Table 5.1: Parameters of populations

\begin{tabular}{|c|c|c|}
\hline Sl. No & Parameters & $\begin{array}{c}\text { Values of the } \\
\text { Parameters }\end{array}$ \\
\hline 1 & $\mathrm{~N}$ & 61 \\
\hline 2 & $\bar{Y}$ & 7.46 \\
\hline 3 & $\bar{X}_{1}$ & 5.31 \\
\hline 4 & $\bar{X}_{2}$ & 179.00 \\
\hline 5 & $\rho_{Y X_{1}}$ & 0.7737 \\
\hline 6 & $\rho_{Y X_{2}}$ & -0.2070 \\
\hline 7 & $\rho_{X_{1} X_{2}}$ & -0.0033 \\
\hline 8 & $C_{y}$ & 0.7103 \\
\hline 9 & $C_{X_{1}}$ & 0.7574 \\
\hline 10 & $C_{X_{2}}$ & 0.2515 \\
\hline 11 & $C_{y}^{2}$ & 0.5046 \\
\hline 12 & $C_{X_{1}}^{2}$ & 0.5737 \\
\hline 13 & $C_{X_{2}}^{2}$ & 0.0633 \\
\hline 14 & $C_{01}$ & 0.4162 \\
\hline 15 & $C_{02}$ & 0.0369 \\
\hline$W_{10 p t}=\frac{-0.0006}{2}+C_{01}+C_{02}+C_{12}$ & \\
\hline$C_{1}^{2}+C_{2}^{2}+2 C_{12}$ & & \\
\hline 16 & $C_{12}$ & \\
\hline Let's further assume that the sample size is 20. \\
\hline
\end{tabular}

$W_{2 o p t}=0.3048300646$ as $W_{1 o p t}+W_{2 o p t}=1$

The biases and MSEs of the competing estimators have been computed and presented in Table 5.2
Table 5.2: Bias and MSE of the competing estimators

\begin{tabular}{|c|c|c|c|}
\hline Sl. No. & $\begin{array}{c}\text { Competing } \\
\text { Estimator }\end{array}$ & $\mid$ Bias $\mid / \boldsymbol{\theta} \bar{Y}$ & $\boldsymbol{M S E} / \boldsymbol{\theta} \overline{\mathbf{Y}}^{\mathbf{2}}$ \\
\hline 1 & $\bar{y}$ & 0.0000 & 0.5045 \\
\hline 2 & $\bar{y}_{R}$ & 0.1574 & 0.2457 \\
\hline 3 & $\bar{y}_{P}$ & -0.0369 & 0.4938 \\
\hline 4 & $\bar{y}_{R P}$ & 0.1211 & 0.2363 \\
\hline 5 & $\bar{y}_{R P}^{*}$ & 0.4131 & 0.1866 \\
\hline
\end{tabular}

Table 5.3PRE of various estimators w.r.t $\bar{y}$

\begin{tabular}{|l|c|l|}
\hline Sl. No. & CompetingEstimator & $\begin{array}{l}\text { Percentage } \\
\text { relative } \\
\text { efficiency } \\
\text { (PRE) }\end{array}$ \\
\hline 1 & $\bar{y}$ & 100 \\
\hline 2 & $\bar{y}_{R}$ & 205.32 \\
\hline 3 & $\bar{y}_{P}$ & 102.16 \\
\hline 4 & $\bar{y}_{R P}$ & 213.49 \\
\hline 5 & $\bar{y}_{R P}^{*}$ & 270.30 \\
\hline
\end{tabular}

From Tables $5.2 \& 5.3$, it is clearly seen that the newly proposed estimator $\bar{y}_{R P}^{*}$ comes out to be the most efficient among all the competing estimators.

\section{CONCLUSION}

The proposed weighted ratio-cum-product estimator has been shown to fare better than its competing estimators, e.g., $\bar{y}, \bar{y}_{R}, \bar{y}_{P}, \bar{y}_{R P}$ under practicalconditions. The theoretical developments have been numerically established. New ratio-cumproduct estimators can be designed using different weighting systems such as weighted geometric mean and weighted harmonic mean and their performance can be evaluated vis-a-vis the existing ratio-cumproduct estimators.

\section{ACKNOWLEDGEMENT}

The authors gratefully acknowledge the suggestions of the reviewers with a view to improving the clarity of presentation.

\section{REFERENCES}

[1] Goodman, L.A., On exact variance of products. Amer. Stat. Asso.55, 708-713, (1960). 
[2] Hansen, M.H. et. al: Sample surveys Methods and Theory. Vol.I Wiley, New York,(1953).

[3] Koop, J.C., "On an identity for the variance of a ratio of two random variables.” Jour. Roy. Stat. Soc. Series B,26(3), 484486, (1964).

[4] Murthy, M.N., Product method of estimation.Sankhya, Series A, Vol.26, 69-74, (1964).

[5] Olkin, I, Multivariate ratio method of estimation for finite populations. Biometrika, 45, 154-65. (1958).

[6] Singh, M.P: On the estimation of ratio and product of population parameters. Sankhya, B, 27,321-28, (1965).

[7] Singh, M.P., Efficient use of systematic sampling in ratio and product estimation, Metrika113, 199-205, (1966).

[8] Singh, M.P., Multivariate product method of estimation for finite populations.,J. Indian Soc. Agri. Statist., 19,1-10, (1967).

[9] Singh, M.P.: Ratio cum Product Method of Estimation, Metrika, Vol.12, Issue 1, pp34-42, (1967).

[10] Singh, M.P., 1969. Comparison of some ratio-cum-product estimators. Sankhya, series B, 31, 375-378.

\section{Author's Profile}

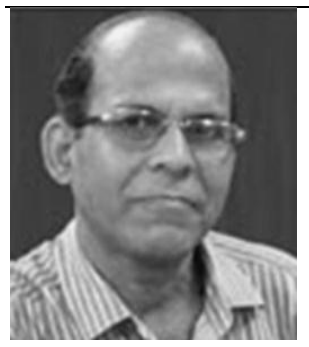

K.B.Panda is Professor of Statistics, Utkal University. He was topper inM.Sc. (Statistics) fromUtkal University and was awarded the Ph.D. degree from Delhi University. His research interest is survey sampling, He has published about 30 research papers in national/international journals and has coauthored a book $\mathrm{He}$ has produced $1 \mathrm{PhD}$ and 5 are presently working.

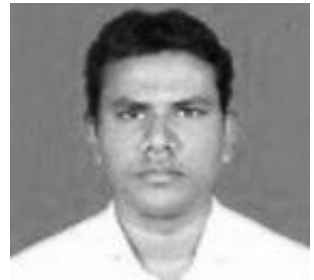

ManoranjanSen, Research Scholar, is pursuing for his $\mathrm{Ph} . \mathrm{D}$. degree in Statistics from Utkal University, Bhubaneswar, Odisha,

Note:Utkal University is an A+ grade University as per NAAC and category 1 University declared by the UGC. It is the premier University of Odisha. 